\title{
ON THE NUMBER OF REPRESENTATIONS OF CERTAIN QUADRATIC FORMS IN 20 AND 24 VARIABLES
}

\author{
B. Ramakrishnan, Brundaban Sahu
}

\begin{abstract}
In this paper, we find the number of representations of certain quadratic forms in 20 and 24 variables. We get this as an application of the evaluation of certain triple convolution sums of the divisor functions. Further, by comparing our formulas with that of Lomadze, we get expressions of certain cusp forms in terms of some finite sums involving the solution set of the quadratic form representation.
\end{abstract}

Keywords: convolution sums of the divisor functions, representation numbers of quadratic forms, modular forms of one variable.

\section{Introduction}

For positive integers $a, b, s, t$, define the convolution sum $W_{a, b}^{s, t}(n)$ by

$$
W_{a, b}^{s, t}(n):=\sum_{\substack{l, m \in \mathbb{N} \\ a l+b m=n}} \sigma_{s}(l) \sigma_{t}(m) .
$$

When $s=t=1$, it is denoted by $W_{a, b}(n)$, and $W_{a, 1}(n)=W_{1, a}(n)$ is denoted by $W_{a}(n)$. These type of sums were evaluated as early as the 19 th century. For example, the sum $W_{1}(n)$ was evaluated by M. Besge, J. W. L. Glaisher and S. Ramanujan $[2,4,14]$. Some of the convlotution sums of the above type have been obtained by several authors (see for example $[5,15,12,17]$ and also the works of K. S. Williams and his co-authors ([16] and the references therein)).

We now define the triple convolution sums of the divisor functions by

$$
W_{a, b, c}^{r, s, t}(n):=\sum_{\substack{l, m, p \in \mathbb{N} \\ a l+b m+c p=n}} \sigma_{r}(l) \sigma_{s}(m) \sigma_{t}(p),
$$

where $a, b, c, r, s, t \in \mathbb{N}$. We write $W_{a, b, c}^{1,1,1}(n)=W_{a, b, c}(n)$ for $a, b, c \in \mathbb{N}$. In [1], Alaca et al. evaluated the convolution sums $W_{1,2,2}(n), W_{1,1,2}(n)$ and $W_{1,2,4}(n)$

2010 Mathematics Subject Classification: primary: 11A25, 11E25; secondary: 11E20, $11 \mathrm{~F} 11$ 
by expressing the product of Eisentein series in terms of their derivatives. In [7, p.11], Kim et al. have treated the convolution sum $W_{1,1,1}(n)$ and as an application, they prove that certain $q$-series satisfy a particular differential equation. Using the theory of modular forms and quasimodular forms, in this article, we evaluate the convolution sums $W_{a, b, c}^{1,3,3}(n)$, where $(a, b, c) \in\{(1,1,1),(1,1,3),(1,3,3),(3,1,1)$, $(3,3,1)\}$ and $W_{a, b, c}^{3,3,3}(n)$, where $(a, b, c) \in\{(1,1,1),(1,1,3),(1,3,3)\}$. As an application, we find formulas for the number of representations of the quadratic forms

$$
F_{k}: x_{1}^{2}+x_{1} x_{2}+x_{2}^{2}+\ldots+x_{2 k-1}^{2}+x_{2 k-1} x_{2 k}+x_{2 k}^{2},
$$

when $k=10,12$. Let

$$
s_{2 k}(n)=\operatorname{card}\left\{\left(x_{1}, x_{2}, \cdots, x_{2 k}\right) \in \mathbb{Z}^{2 k}: F_{k}\left(x_{1}, x_{2}, \cdots, x_{2 k}\right)=n\right\}
$$

be the number of representations of a positive integer $n$ by the quadratic form $F_{k}$. For $k=2,4,6,8$ formulas for $s_{2 k}$ are known due to the works of J. Liouville [9], J. G. Huard et al. [5], O. X. M. Yao and E. X. W. Xia [17] and the authors [13]. In [10], G. A. Lomadze gave formulas for $s_{2 k}(n)$ for $2 \leqslant n \leqslant 17$, which involves the divisor functions and certain finite sums which involve the solution set of the representation of same quadratic forms of lower variables. However, the other formulas mentioned above are in terms of divisor functions and Fourier coefficients of certain cusp forms. Like in the works of [17] and [13], by comparing the formulas of Lomadze with our results, we also obtain identities connecting the Fourier coefficients of certain cusp forms in terms of finite sums (see Corollary 2.5).

\section{Preliminaries and statement of the results}

Let $M_{k}(N)$ be the space of modular forms of weight $k$ for the congruence subgroup $\Gamma_{0}(N)$ and $S_{k}(N)$ be the subspace of cusp forms of weight $k$ for the congruence subgroup $\Gamma_{0}(N)$. For $k \geqslant 4$, let $E_{k}$ denote the normalized Eisenstein series of weight $k$ in $M_{k}(1)$ given by

$$
E_{k}(z)=1-\frac{2 k}{B_{k}} \sum_{n \geqslant 1} \sigma_{k-1}(n) q^{n},
$$

where $q=e^{2 i \pi z}$ and $B_{k}$ is the $k$-th Bernoulli number defined by

$$
\frac{x}{e^{x}-1}=\sum_{m=0}^{\infty} \frac{B_{m}}{m !} x^{m} .
$$

The first few Eisenstein series are given as follows:

$$
\begin{aligned}
& E_{4}(z)=1+240 \sum_{n \geqslant 1} \sigma_{3}(n) q^{n}, \quad E_{6}(z)=1-504 \sum_{n \geqslant 1} \sigma_{5}(n) q^{n} \\
& E_{8}(z)=1+480 \sum_{n \geqslant 1} \sigma_{7}(n) q^{n}, \quad E_{10}(z)=1-264 \sum_{n \geqslant 1} \sigma_{9}(n) q^{n} \\
& E_{12}(z)=1+\frac{65520}{691} \sum_{n \geqslant 1} \sigma_{11}(n) q^{n} .
\end{aligned}
$$


The following identity is well-known from the fact that $E_{8}=E_{4}^{2}$ :

$$
W_{1,1}^{3,3}(n)=\frac{1}{120} \sigma_{7}(n)-\frac{1}{120} \sigma_{3}(n)
$$

In order to evaluate the convolutions sums $W_{a, b, c}^{1, s, t}(n)$, we use the structure theorem on quasimodular forms of weight $k$ and depth $\leqslant k / 2$. For details on basics of modular forms and quasimodular forms, we refer the reader to $[3,6,11]$. The Eisenstein series $E_{2}$, which is a quasimodular form of weight 2 , depth 1 on $S L_{2}(\mathbb{Z})$ is given by

$$
E_{2}(z)=1-24 \sum_{n \geqslant 1} \sigma(n) e^{2 \pi i n z}
$$

and this fundamental quasimodular form will be used in our results. The space of quasimodular forms of weight $k$, depth $\leqslant k / 2$ on $\Gamma_{0}(N)$ is denoted by $\tilde{M}_{k}^{\leqslant k / 2}(N)$. We need the following structure theorem (see $[6,11])$. For an even integer $k$ with $k \geqslant 2$, we have

$$
\tilde{M}_{k}^{\leqslant k / 2}(N)=\bigoplus_{j=0}^{k / 2-1} D^{j} M_{k-2 j}(N) \oplus \mathbb{C} D^{k / 2-1} E_{2}
$$

where the differential operator $D$ is defined by $D:=\frac{1}{2 \pi i} \frac{d}{d z}$. Using this one can express each quasimodular form of weight $k$ and depth $\leqslant k / 2$ as a linear combination of $j$-th derivatives of modular forms of weight $k-2 j$ on $\Gamma_{0}(N), 0 \leqslant j \leqslant k / 2-1$ and the $(k / 2-1)$-th derivate of the quasimodular form $E_{2}$.

We need the following newforms for our results. Let $\Delta(z)=\sum_{n \geqslant 1} \tau(n) q^{n}=$ $\eta^{24}(z)$ be the well-known unique normalized cusp form of weight 12 , level 1 , studied by Ramanujan. Here $\eta(z)$ is the Dedekind eta function given by

$$
\eta(z)=q^{1 / 24} \prod_{n \geqslant 1}\left(1-q^{n}\right)
$$

Let $\left\{\Delta_{k, N, j}: 1 \leqslant j \leqslant d\right\}$ be the basis (of dimension $d$ ) of normalized newforms of weight $k$, level $N$, having Fourier expansion

$$
\Delta_{k, N, j}(z)=\sum_{n \geqslant 1} \tau_{k, N, j}(n) q^{n}
$$

If $d=1$, then we write the function as $\Delta_{k, N}$ and its Fourier coefficients as $\tau_{k, N}(n)$. 
The following are the main theorems of this section.

Theorem 2.1. Let $n \in \mathbb{N}$, then

$$
\begin{aligned}
& W_{1,1,1}^{1,3,3}(n)=\frac{1}{240^{2}}\left[11 \sigma_{9}(n)+10(2-3 n) \sigma_{7}(n)-42 \sigma_{5}(n)\right. \\
& \left.+20(3 n-1) \sigma_{3}(n)+\sigma(n)\right], \\
& W_{1,1,3}^{1,3,3}(n)=\frac{1}{240^{2}}\left[\frac{91}{671} \sigma_{9}(n)+\frac{7290}{7381} \sigma_{9}\left(\frac{n}{3}\right)-\frac{15}{41} n \sigma_{7}(n)-\frac{1215}{41} n \sigma_{7}\left(\frac{n}{3}\right)\right. \\
& +\frac{10}{41} \sigma_{7}(n)+\frac{810}{41} \sigma_{7}\left(\frac{n}{3}\right)-\frac{276}{13} \sigma_{5}(n)-\frac{270}{13} \sigma_{5}\left(\frac{n}{3}\right)+30 n \sigma_{3}(n) \\
& +30 n \sigma_{3}\left(\frac{n}{3}\right)-10 \sigma_{3}(n)+10 \sigma_{3}\left(\frac{n}{3}\right)+\sigma(n)-\frac{280}{61} \tau_{10,3,1}(n) \\
& \left.+115 \tau_{10,3,2}(n)-\frac{600}{41} n \tau_{8,3}(n)+\frac{400}{41} \tau_{8,3}(n)-\frac{10}{13} \tau_{6,3}(n)\right], \\
& W_{1,3,3}^{1,3,3}(n)=\frac{1}{240^{2}}\left[\sigma(n)-20(1-3 n) \sigma_{3}\left(\frac{n}{3}\right)-\frac{6}{13} \sigma_{5}(n)-\frac{540}{13} \sigma_{5}\left(\frac{n}{3}\right)\right. \\
& -\frac{20}{13} \tau_{6,3}(n)+10(2-3 n) \sigma_{7}\left(\frac{n}{3}\right)+\frac{11}{7381} \sigma_{9}(n)+\frac{7380}{671} \sigma_{9}\left(\frac{n}{3}\right) \\
& \left.+\frac{160}{549} \tau_{10,3,1}(n)+\frac{70}{99} \tau_{10,3,2}(n)\right] \text {, } \\
& W_{3,1,1}^{1,3,3}(n)=\frac{1}{240^{2}}\left[\sigma\left(\frac{n}{3}\right)+20(n-1) \sigma_{3}(n)-\frac{60}{13} \sigma_{5}(n)-\frac{486}{13} \sigma_{5}\left(\frac{n}{3}\right)\right. \\
& +\frac{60}{13} \tau_{6,3}(n)+10(2-n) \sigma_{7}(n)+\frac{890}{671} \sigma_{9}(n)+\frac{6561}{671} \sigma_{9}\left(\frac{n}{3}\right) \\
& \left.+\frac{480}{61} \tau_{10,3,1}(n)-\frac{210}{11} \tau_{10,3,2}(n)\right], \\
& W_{3,3,1}^{1,3,3}(n)=\frac{1}{240^{2}}\left[\sigma\left(\frac{n}{3}\right)+10(n-1) \sigma_{3}\left(\frac{n}{3}\right)+10(n-1) \sigma_{3}(n)-\frac{516}{13} \sigma_{5}\left(\frac{n}{3}\right)\right. \\
& -\frac{30}{13} \sigma_{5}(n)+\frac{30}{13} \tau_{6,3}(n)+\frac{10-5 n}{41} \sigma_{7}(n)+\frac{810-405 n}{41} \sigma_{7}\left(\frac{n}{3}\right) \\
& +\frac{400}{41} \tau_{8,3}(n)+\frac{10}{671} \sigma_{9}(n)+\frac{7371}{671} \sigma_{9}\left(\frac{n}{3}\right)-\frac{280}{183} \tau_{10,3,1}(n) \\
& \left.-\frac{115}{33} \tau_{10,3,2}(n)-\frac{200}{41} n \tau_{8,3}(n)\right] \text {. }
\end{aligned}
$$


Theorem 2.2. Let $n \in \mathbb{N}$, then

$$
\begin{aligned}
W_{1,1,1}^{3,3,3}(n)= & \frac{1}{19200} \sigma_{3}(n)-\frac{1}{9600} \sigma_{7}(n)+\frac{91}{13267200} \sigma_{11}(n)+\frac{1}{22112} \tau(n), \\
W_{1,1,3}^{3,3,3}(n)= & \frac{41}{484252800} \sigma_{11}(n)+\frac{6561}{968505600} \sigma_{11}\left(\frac{n}{3}\right)-\frac{7}{196800} \sigma_{7}(n) \\
& -\frac{9}{131200} \sigma_{7}\left(\frac{n}{3}\right)+\frac{1}{28800} \sigma_{3}(n)+\frac{1}{57600} \sigma_{3}\left(\frac{n}{3}\right)-\frac{133}{720 \times 11747} \tau(n) \\
& +\frac{145071}{160 \times 11747} \tau\left(\frac{n}{3}\right)-\frac{1}{29520} \tau_{8,3}(n)+\frac{1}{16 \times 1241} \tau_{12,3}(n), \\
W_{1,3,3}^{3,3,3}(n)= & \frac{91}{19200 \times 691 \times 6643} \sigma_{11}(n)+\frac{91 \times 6642}{19200 \times 691 \times 6643} \sigma_{11}\left(\frac{n}{3}\right) \\
& -\frac{1}{1180800} \sigma_{7}(n)-\frac{61}{590400} \sigma_{7}\left(\frac{n}{3}\right)+\frac{1}{240^{2}} \sigma_{3}(n)+\frac{1}{28800} \sigma_{3}\left(\frac{n}{3}\right) \\
& +\frac{199}{1440 \times 11747} \tau(n)-\frac{1197}{80 \times 11747} \tau\left(\frac{n}{3}\right)-\frac{1}{120 \times 246} \tau_{8,3}(n) \\
& +\frac{1}{144 \times 1241} \tau_{12,3}(n) .
\end{aligned}
$$

We apply the above convolution sums to derive the following theorems.

Theorem 2.3. The number of representations of a positive integer $n$ by the quadratic form $F_{10}$ is given by

$$
s_{20}(n)=\frac{12}{11} \sigma_{9}^{*}(n)+\frac{648}{11} \tau_{10,3,2}(n)
$$

where $\sigma_{9}^{*}(n)=\sigma_{9}(n)-3^{5} \sigma_{9}\left(\frac{n}{3}\right)$.

Theorem 2.4. The number of representations of a positive integer $n$ by the quadratic form $F_{12}$ is given by

$$
s_{24}(n)=\frac{6552}{50443} \sigma_{11}^{*}(n)+\frac{402624}{11747} \tau(n)+\frac{293512896}{11747} \tau\left(\frac{n}{3}\right)+\frac{46656}{1241} \tau_{12,3}(n),
$$

where $\sigma_{11}^{*}(n)=\sigma_{11}(n)+3^{6} \sigma_{11}\left(\frac{n}{3}\right)$.

Corollary 2.5. Comparing our formulas in Theorem 2.3 and Theorem 2.4 with the formulas (IX) and (XI) in p. 12 of [10], we get the following identities:

$$
\tau_{10,3,2}(n)=\frac{1}{120} \sum_{F_{6}\left(x_{1}, \cdots, x_{12}\right)=n}\left(42 x_{1}^{4}-27 n x_{1}^{2}+n^{2}\right),
$$




$$
\begin{aligned}
\frac{402624}{11747} & \tau(n)+\frac{293512896}{11747} \tau\left(\frac{n}{3}\right)+\frac{46656}{1241} \tau_{12,3}(n) \\
= & \frac{291096}{1765505} \sum_{F_{8}\left(x_{1}, \cdots, x_{16}\right)=n}\left(135 x_{1}^{4}-54 n x_{1}^{2}+2 n^{2}\right) \\
& +\frac{864}{50443} \sum_{F_{6}\left(x_{1}, \cdots, x_{12}\right)=n}\left(162 x_{1}^{6}-162 n x_{1}^{4}+36 n^{2} x_{1}^{2}-n^{3}\right) \\
& +\frac{30}{50443} \sum_{F_{4}\left(x_{1}, \cdots, x_{8}\right)=n}\left(1215 x_{1}^{8}-2268 n x_{1}^{6}+1260 n^{2} x_{1}^{4}-210 n^{3} x_{1}^{2}+5 n^{4}\right) .
\end{aligned}
$$

Remark 2.1. It would be interesting to get individual expressions for the cusp forms appearing in (7), which will give an explicit expression for the Ramanujan Tau function.

\section{Proofs}

For the proofs of our theorems, we need the newforms $\Delta_{k, N}(z),(k, N) \in\{(6,3)$, $(8,3),(12,3)\}, \Delta_{10,3,1}(z), \Delta_{10,3,2}(z)$. Below we give their expressions in terms of Eisenstein series and eta products. We have used the L-functions and modular forms database [8] to get these expressions. (The expression for $\Delta_{8,3}(z)$ appeared in $[13$, Eq.(10)].)

$$
\begin{aligned}
\Delta_{6,3}(z) & =\eta^{6}(z) \eta^{6}(3 z), \\
\Delta_{8,3}(z) & =\eta^{12}(z) \eta^{4}(3 z)+81 \eta^{6}(z) \eta^{4}(3 z) \eta^{6}(9 z)+18 \eta^{9}(z) \eta^{4}(3 z) \eta^{3}(9 z), \\
\Delta_{10,3,1}(z) & =\frac{-1}{8} E_{4}(z) \Delta_{6,3}(z)+\frac{9}{8} E_{4}(3 z) \Delta_{6,3}(z), \\
\Delta_{10,3,2}(z) & =\frac{1}{10} E_{4}(z) \Delta_{6,3}(z)+\frac{9}{10} E_{4}(3 z) \Delta_{6,3}(z), \\
\Delta_{12,3}(z) & =\frac{98}{81} \Delta(z)-3402 \Delta(3 z)-\frac{17}{81} E_{6}(z) \Delta_{6,3}(z) .
\end{aligned}
$$

\subsection{Proof of Theorem 2.1}

We need the following convolution sums (see $[13,17])$.

Proposition 3.1. Let $n \in \mathbb{N}$. Then

$$
\begin{aligned}
& W_{1,3}^{3,3}(n)=-\frac{1}{240} \sigma_{3}(n)-\frac{1}{240} \sigma_{3}\left(\frac{n}{3}\right)+\frac{1}{9840} \sigma_{7}(n)+\frac{81}{9840} \sigma_{7}\left(\frac{n}{3}\right)+\frac{1}{246} \tau_{8,3}(n), \\
& W_{1,3}^{1,1}(n)=\frac{7}{80} \sigma_{5}(n)-\frac{1}{8} n \sigma_{3}(n)+\frac{1}{24} \sigma_{3}(n)-\frac{1}{240} \sigma(n), \\
& W_{3,1}^{1,3}(n)=\frac{1}{104} \sigma_{5}(n)-\frac{81}{1040} \sigma_{5}\left(\frac{n}{3}\right)+\frac{1-n}{24} \sigma_{3}(n)-\frac{1}{240} \sigma\left(\frac{n}{3}\right)-\frac{1}{104} \tau_{6,3}(n), \\
& W_{1,3}^{1,3}(n)=\frac{1}{1040} \sigma_{5}(n)+\frac{9}{104} \sigma_{5}\left(\frac{n}{3}\right)+\frac{1-3 n}{24} \sigma_{3}\left(\frac{n}{3}\right)-\frac{1}{240} \sigma(n)+\frac{1}{312} \tau_{6,3}(n) .
\end{aligned}
$$


The vector space $M_{10}(3)$ is of dimension 4 with a basis $\left\{E_{10}(z), E_{10}(3 z), \Delta_{10,3,1}(z)\right.$, $\left.\Delta_{10,3,2}(z)\right\}$, the vector space $M_{8}(3)$ is of dimension 3 with a basis $\left\{E_{8}(z), E_{8}(3 z)\right.$, $\left.\Delta_{8,3}(z)\right\}$, the vector space $M_{6}(3)$ is of dimension 3 with a basis $\left\{E_{6}(z), E_{6}(3 z)\right.$, $\left.\Delta_{6,3}(z)\right\}$, and the space $M_{4}(3)$ has dimension 2 with a basis $\left\{E_{4}(z), E_{4}(3 z)\right\}$. Now using the structure theorem of quasimodular forms and using the above basis, we get the following.

$$
\begin{aligned}
E_{2}(z) E_{4}^{2}(z)= & E_{10}(z)+\frac{3}{2} D E_{8}(z) \\
E_{2}(z) E_{4}(z) E_{4}(3 z)= & \frac{91}{7381} E_{10}(z)+\frac{7290}{7381} E_{10}(3 z)+\frac{6720}{61} \Delta_{10,3,1}(z) \\
& -\frac{2760}{11} \Delta_{10,3,2}(z)+\frac{3}{164} D E_{8}(z)+\frac{243}{164} D E_{8}(3 z) \\
& +\frac{14400}{41} D \Delta_{8,3}(z), \\
E_{2}(z) E_{4}^{2}(3 z)= & \frac{1}{7381} E_{10}(z)+\frac{7380}{7381} E_{10}(3 z)-\frac{1280}{183} \Delta_{10,3,1}(z) \\
& -\frac{560}{33} \Delta_{10,3,2}(z)+\frac{3}{2} D E_{8}(3 z), \\
E_{2}(3 z) E_{4}^{2}(z)= & \frac{820}{7381} E_{10}(z)+\frac{6561}{7381} E_{10}(3 z)-\frac{11520}{61} \Delta_{10,3,1}(z) \\
& +\frac{5040}{11} \Delta_{10,3,2}(z)+\frac{1}{2} D E_{8}(z), \\
E_{2}(3 z) E_{4}(z) E_{4}(3 z)= & \frac{10}{7381} E_{10}(z)+\frac{7371}{7381} E_{10}(3 z)+\frac{2240}{61} \Delta_{10,3,1}(z) \\
& +\frac{920}{11} \Delta_{10,3,2}(z)+\frac{1}{164} D E_{8}(z)+\frac{81}{164} D E_{8}(3 z) \\
& +\frac{4800}{41} D \Delta_{8,3}(z) .
\end{aligned}
$$

By comparing the $n$-th Fourier coefficients and using the convolution sums $W_{1,1}^{3,3}, W_{1,3}^{3,3}, W_{1,1}^{1,3}, W_{1,3}^{1,3}$ from Proposition 3.1 we get the required triple convolution sums.

\subsection{Proof of Theorem 2.2}

The vector space $M_{12}(1)$ has dimension 2 with a basis $\left\{E_{12}(z), \Delta(z)\right\}$, where $\Delta(z)$ is the unique normalized newform of weight 12 and level 1 . Now $E_{4}^{3}(z) \in M_{12}(1)$ and writing as linear combination of basis, we have

$$
E_{4}^{3}(z)=E_{12}(z)+\frac{432000}{691} \Delta(z)
$$

The dimension of the space $M_{12}(3)$ is 5 having a basis $\left\{E_{12}(z), E_{12}(3 z), \Delta(z)\right.$, $\left.\Delta(3 z), \Delta_{12,3}(z)\right\}$, where $\Delta_{12,3}(z)$ is the unique normalized newform of weight 12 and level 3 . 
Now $E_{4}^{2}(z) E_{4}(3 z), E_{4}(z) E_{4}^{2}(3 z) \in M_{12}(3)$. Writing as linear combination of the above basis, we get

$$
\begin{aligned}
E_{4}^{2}(z) E_{4}(3 z)= & \frac{82}{6643} E_{12}(z)+\frac{6561}{6643} E_{12}(3 z)-\frac{2553600}{11747} \Delta(z) \\
& +\frac{12534134400}{11747} \Delta(3 z)+\frac{86400}{1241} \Delta_{12,3}(z)
\end{aligned}
$$

and

$$
\begin{aligned}
E_{4}(z) E_{4}^{2}(3 z)= & \frac{1}{6643} E_{12}(z)+\frac{6642}{6643} E_{12}(3 z)+\frac{1910400}{11747} \Delta(z) \\
& -\frac{206841600}{11747} \Delta(3 z)+\frac{96000}{1241} \Delta_{12,3}(z) .
\end{aligned}
$$

By comparing the $n$-th Fourier coefficients and using convolution sums $W_{1,1}^{3,3}$ from (4) and $W_{1,3}^{3,3}$ from Proposition 3.1 we get the required convolution sums.

\subsection{Proof of Theorem 2.3}

Let $\mathbb{N}_{0}=\mathbb{N} \cup\{0\}$. For $n \in N$ we know that (see [5], [10])

$$
s_{4}(n)=12 \sigma(n)-36 \sigma\left(\frac{n}{3}\right),
$$

and

$$
s_{8}(n)=24 \sigma_{3}(n)+216 \sigma_{3}\left(\frac{n}{3}\right) .
$$

Then $s_{20}(n)$ is given by

$$
\begin{aligned}
s_{20}(n)= & \sum_{\substack{a, b, c \in \mathbb{N}_{0} \\
a+b+c=n}}\left(\sum_{F_{2}\left(x_{1}, \cdots, x_{4}\right)=a} 1\right)\left(\sum_{F_{4}\left(x_{5}, \cdots, x_{12}\right)=b} 1\right)\left(\sum_{F_{4}\left(x_{13}, \cdots, x_{20}\right)=c} 1\right) \\
= & s_{4}(n)+2 s_{8}(n)+\sum_{\substack{a, b \in \mathbb{N} \\
a+b=n}} s_{8}(a) s_{8}(b)+2 \sum_{\substack{a, b \in \mathbb{N} \\
a+b=n}} s_{4}(a) s_{8}(b) \\
& +\sum_{\substack{a, b, c \in \mathbb{N} \\
a+b+c=n}} s_{4}(a) s_{8}(b) r_{8}(c) \\
= & 12 \sigma(n)-36 \sigma\left(\frac{n}{3}\right)+48 \sigma_{3}(n)+432 \sigma_{3}\left(\frac{n}{3}\right)+24^{2} W_{1,1}^{3,3}+48 \times 216 W_{1,3}^{3,3} \\
& +216^{2} W_{1,1}^{3,3}\left(\frac{n}{3}\right)+24^{2} W_{1,1}^{1,3}+9 \times 24^{2} W_{1,3}^{1,3}-48 \times 36 W_{3,1}^{1,3} \\
& -36 \times 216 W_{1,1}^{1,3}\left(\frac{n}{3}\right)+12 \times 24^{2} W_{1,1,1}^{1,3,3}+9 \times 24^{3} W_{1,1,3}^{1,3,3} \\
& +12 \times 216^{2} W_{1,3,3}^{1,3,3}-36 \times 24^{2} W_{3,1,1}^{1,3,3}-72^{3} W_{3,1,3}^{1,3,3}-1296^{2} W_{1,1,1}^{1,3,3}\left(\frac{n}{3}\right) .
\end{aligned}
$$

Now, we substitute the expressions for the covolution sums using (4) and Theorem 2.1 , the required formula follows. 


\subsection{Proof of Theorem 2.4}

We proceed as in the case of 20 variables. We have

$$
\begin{aligned}
s_{24}(n)= & \sum_{\substack{a, b, c \in \mathbb{N}_{0} \\
a+b+c=n}}\left(\sum_{F_{4}\left(x_{1}, \cdots, x_{8}\right)=a} 1\right)\left(\sum_{F_{4}\left(x_{9}, \cdots, x_{16}\right)=b} 1\right)\left(\sum_{F_{4}\left(x_{17}, \cdots, x_{24}\right)=c} 1\right) \\
= & 3 s_{8}(n)+3 \sum_{\substack{a, b \in \mathbb{N} \\
a+b=n}} s_{8}(a) s_{8}(b)+\sum_{\substack{a, b, c \in \mathbb{N} \\
a+b+c=n}} s_{8}(a) s_{8}(b) s_{8}(c) \\
= & 72 \sigma_{3}(n)+648 \sigma_{3}\left(\frac{n}{3}\right)+3 \sum_{\substack{a, b \in \mathbb{N} \\
a+b=n}}\left(24 \sigma_{3}(a)+216 \sigma_{3}\left(\frac{a}{3}\right)\right) \\
& \times\left(24 \sigma_{3}(b)+216 \sigma_{3}\left(\frac{b}{3}\right)\right)+\sum_{\substack{a, b, c \in \mathbb{N} \\
a+b+c=n}}\left(24 \sigma_{3}(a)+216 \sigma_{3}\left(\frac{a}{3}\right)\right) \\
& \times\left(24 \sigma_{3}(b)+216 \sigma_{3}\left(\frac{b}{3}\right)\right)\left(24 \sigma_{3}(c)+216 \sigma_{3}\left(\frac{c}{3}\right)\right) \\
= & 72 \sigma_{3}(n)+648 \sigma_{3}\left(\frac{n}{3}\right)+3 \times 24^{2} W_{1,1}^{3,3}(n)+54 \times 24^{2} W_{1,3}^{3,3}(n) \\
& +3^{5} \times 24^{2} W_{1,1}^{3,3}\left(\frac{n}{3}\right)+24^{3} W_{1,1,1}^{3,3,3}(n)+3^{3} \times 24^{3} W_{1,1,3}^{3,3,3}(n) \\
& +3^{5} \times 24^{3} W_{1,3,3}^{3,3,3}(n)+216^{3} W_{1,1,1}^{3,3,3}\left(\frac{n}{3}\right) .
\end{aligned}
$$

Substituting the convolution sums using (4), Proposition 3.1 and Theorem 2.2, we get the required formula for $s_{24}(n)$.

We give below a table giving the first 15 values of $s_{10}(n)$ and $s_{24}(n)$.

\begin{tabular}{|r|r|r|}
\hline$n$ & \multicolumn{1}{|c|}{$s_{20}(n)$} & \multicolumn{1}{|c|}{$s_{24}(n)$} \\
\hline 1 & 60 & 72 \\
2 & 1620 & 2376 \\
3 & 25980 & 47592 \\
4 & 275460 & 646344 \\
5 & 2040552 & 6305904 \\
6 & 10965780 & 45821160 \\
7 & 44559840 & 255215808 \\
8 & 145963620 & 1125009864 \\
9 & 417830460 & 4097478600 \\
10 & 1091417976 & 12975540336 \\
11 & 2573551440 & 37101202848 \\
12 & 5569628100 & 96867424872 \\
13 & 11570383560 & 232791251760 \\
14 & 22593025440 & 526183909056 \\
15 & 41415305832 & 1128351033648 \\
\hline
\end{tabular}


Acknowledgements. We have used the open-source mathematics software SAGE (www.sagemath.org) to do our calculations. The second author is partially funded by SERB grant SR/FTP/MS-053/2012. He would like to thank HRI, Allahabad for the warm hospitality where this work has been carried out. Finally, the authors thank the referee for his/her comments and also for pointing out the reference [7].

\section{References}

[1] S. Alaca, F. Uygul and K.S. Williams, Some arithmetic identities involving divisor functions, Funct. Approx. Comment. Math. 46 (2012), no. 2, 261-271.

[2] M. Besge, Extrait d'une lettre de M. Besge á M. Liouville, J. Math. Pures Appl. 7 (1862), 256.

[3] J.H. Bruinier, G. van der Geer, G. Harder and D. Zagier, The 1-2-3 of modular forms, Lectures from the Summer School on Modular Forms and their Applications held in Nordfjordeid, June 2004. Edited by Kristian Ranestad. Universitext. Springer-Verlag, Berlin, 2008. 266 pp.

[4] J.W.L. Glaisher, On the squares of the series in which the coefficients are the sums of the divisor of the exponents, Mess. Math. 15 (1885), 1-20.

[5] J.G. Huard, Z.M. Ou, B.K. Spearman and K.S. Williams, Elementary evaluation of certain convolution sums involving divisor functions, in Number Theory for the Millennium, II (Urbana, IL, 2000) (A. K. Peters, Natick, MA, 2002), 229-274.

[6] M. Kaneko and D. Zagier, A generalized Jacobi theta function and quasimodular forms. In "The moduli space of curves (Texel Island, 1994)", 165-172, Progr. Math. 129, Birkhäuser Boston, Boston, MA, 1995.

[7] D. Kim, A. Kim and A. Sankaranarayanan, Bernoulli numbers, convolution sums and congruences of coefficients for certain generating functions, J. Inequal. Appl. 2013:225 (2013), 26 pp.

[8] LMFDB, The database of L-functions, modular forms, and related objects, http://www.lmfdb.org/

[9] J. Liouville, Sur la formes $x^{2}+x y+y^{2}+z^{2}+z t+t^{2}$, J. Math. Pures. Appl. 8 (1863), 141-144.

[10] G.A. Lomadze, Representation of numbers by sums of the quadratic forms $x_{1}^{2}+x_{1} x_{2}+x_{2}^{2}$, Acta Arith. 54 (1989), 9-36. (in Russian)

[11] F. Martin and E. Royer, Formes modulaires et périodes, In 'Formes modulaires et transcendance', 1-117, Sémin. Congr., 12, Soc. Math. France, Paris, 2005.

[12] B. Ramakrishnan and B. Sahu, Evaluation of the convolution sums $\sum_{l+15 m=n} \sigma(l) \sigma(m)$ and $\sum_{3 l+5 m=n} \sigma(l) \sigma(m)$ and an application, Int. J. Number Theory 9 (2013), no. 3, 1-11.

[13] B. Ramakrishnan and B. Sahu, On the number of representations of an integer by certain quadratic forms in sixteen variables, Int. J. Number Theory 10 (2014), no. 8, 1929-1937.

[14] S. Ramanujan, On certain arithmetical functions, Trans. Cambridge Philos. Soc. 22 (1916) 159-184. 
[15] E. Royer, Evaluating convolution sums of the divisor function by quasimodular forms, Int. J. Number Theory 3 (2007), no. 2, 231-261.

[16] K.S. Williams, Number Theory in the spirit of Liouville, London Mathematical Student Texts 76, Cambridge Univ. Press, 2011.

[17] O.X.M. Yao and E.X.W. Xia, Evaluation of the convolution sum $\sum_{i+3 j=n} \sigma(i) \sigma_{3}(j)$ and $\sum_{3 i+j=n} \sigma(i) \sigma_{3}(j)$, Int. J. Number Theory $10(2014)$, no. $1,115-123$.

Addresses: B. Ramakrishnan: Harish-Chandra Research Institute, Chhatnag Road, Jhunsi, Allahabad - 211 019, India.;

Brundaban Sahu: School of Mathematical Sciences, National Institute of Science Education and Research, PO: Sainik School, Bhubaneswar, Odisha - 751 005, India.

E-mail: ramki@hri.res.in, brundaban.sahu@niser.ac.in

Received: 9 July 2015; revised: 17 September 2015 